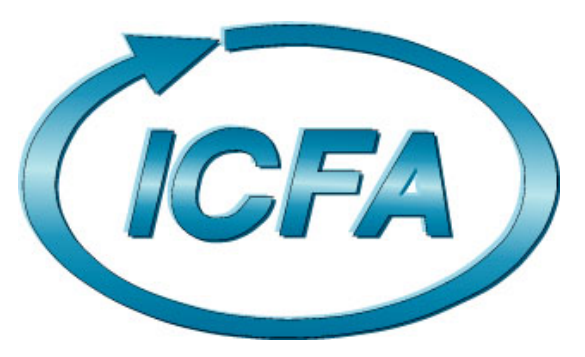

International Committee for Future Accelerators

Sponsored by the Particles and Fields Commission of IUPAP

\title{
Beam Dynamics Newsletter
}

No. 45

Issue Editor:
R. Wanzenberg

Editor in Chief:

W. Chou

April 2008 
22. A.K. Bandyopadhyay, A. Joestingmeier, A.S. Omar, K. Balewski, R. Wanzenberg, "Computation of Wakefields and Impedances for the PETRA III Longitudinal Feedback Cavity”, Asian Particle Accelerator Conference, APAC 07, Indore, India, Jan 29-Feb 02, 2007

23. A.K. Bandyopadhyay, A. Joestingmeier, A.S. Omar, R. Wanzenberg, "Wakes and Impedance Computations for the PETRA III Longitudinal Feedback Cavity”, Internal Report, DESY-M-07-02, Nov 2007

24. M. Ivanyan, E. Laziev, V. Tsakanov, A. Vardanyan, A. Tsakanian, R. Wanzenberg, "Investigation of the PETRA III Resistive Wall Impedance, Intermediate Report of the PETRA III - CANDLE COLLABORATION", Internal Report, DESY-M-07-01, Sep 2007

25. K. Balewski, E. Gjonaj, T. Weiland, , R. Wanzenberg, "Wake Computations for Undulator Vacuum Chambers of PETRA III", Particle Accelerator Conference, PAC07, Albuquerque, New Mexico, 25 to 29 June, 2007

\title{
4.8 PETRA III Storage Ring Resistive Wall Impedance
}

\author{
M. Ivanyan, E. Laziev, V. Tsakanov, A. Vardanyan \\ CANDLE, Acharyan 31, 375040 Yerevan, Armenia \\ Mail to: ivanian@asls.candle.am \\ A. Tsakanian, Hamburg University, Hamburg, Germany \\ Mail to: tsakania@mail.desy.de \\ R. Wanzenberg, DESY, Notkestr. 85, Hamburg, Germany \\ Mail to: rainer.wanzenberg@desy.de
}

\subsubsection{Introduction}

The article presents the results of the resistive wall impedance study for the PETRA III storage ring [1] vacuum chamber that consists of different parts with various geometrical (circular and elliptical cross sections) and structural (single layer and laminated) configurations conditioned by the technical solutions for high brightness synchrotron light sources.

The study is based on the impedance calculation for a laminated vacuum chamber with circular-cylindrical geometry using the field matching technique [2,3]. The field transformation matrix concept is introduced to evaluate analytically the longitudinal and transverse impedances for a layered vacuum chamber with arbitrary materials and thicknesses [4,5]. The results are applied to calculate the impedances for various parts of the vacuum chamber listed in Table 1: the finite thickness standard vacuum chamber, the metallic wiggler chamber with NEG coating and the injection kicker ceramic metallic vacuum chamber. Impedances are evaluated for chambers with circular and elliptical cross sections. Horizontal and vertical geometrical correction factors [6] are used for the impedances calculations of vacuum chambers with elliptical cross section. The kick factor and the integrated gradient of the longitudinal wake potential are also given. 
Table 1: The main parameters of PETRA III vacuum chambers.

\begin{tabular}{|c|c|c|c|c|c|}
\hline $\begin{array}{c}\text { Vacuum } \\
\text { Chamber }\end{array}$ & Material & $\begin{array}{l}\text { Cross- } \\
\text { section }\end{array}$ & $\begin{array}{l}\text { Aperture } \\
(\mathrm{mm})\end{array}$ & $\begin{array}{l}\text { Wall thick- } \\
\text { ness }(\mathrm{mm})\end{array}$ & $\begin{array}{l}\text { Conductivity } \\
10^{6}(\Omega \mathrm{m})^{-1}\end{array}$ \\
\hline $\begin{array}{l}\text { Standard } \\
\text { straight section }\end{array}$ & $\begin{array}{l}\text { Stainless } \\
\text { Steel }\end{array}$ & Circular & 47 & 2 & 1.5 \\
\hline Standard arc & Aluminium & Elliptical & $40 \times 20$ & 4 & 36.6 \\
\hline Undulators & Aluminium & Elliptical & $28.5 \times 3.5$ & 1 & 36.6 \\
\hline Wigglers & $\begin{array}{l}\text { Aluminium } \\
\text { + NEG }\end{array}$ & Elliptical & $48 \times 8.95$ & 2.8 & $\begin{array}{l}36.6 \\
0.31\end{array}$ \\
\hline Injection Kicker & $\begin{array}{l}\text { Ceramic } \\
+ \text { TSHGS }\end{array}$ & Circular & 90 & 10 & $\begin{array}{c}- \\
2.084\end{array}$ \\
\hline
\end{tabular}

\subsubsection{Resistive Impedance of Multi-Layer Vacuum Chamber}

The longitudinal $Z_{\|}(\omega, r, \theta)$ and transverse $\vec{Z}_{\perp}(\omega, r, \theta)$ coupling impedances per unit length are defined as a Fourier transformation of the normalized longitudinal and transverse components of the integrated Lorentz force of the electromagnetic fields, induced by the point charge $q$ moving with constant velocity $v$ along the tube axis $z$. The $m$ - pole azimuthal components of the impedances in ultra-relativistic approximation are given by [2,3]

$$
\begin{aligned}
& Z_{\|}^{(m)}(\omega, r, \theta)=\tilde{Z}_{\|}^{(m)}(k)\left(\frac{r}{a_{1}}\right)^{m}\left(\frac{r_{q}}{a_{1}}\right)^{m} \cos m \theta \\
& \left.\begin{array}{l}
Z_{r}^{(m)} \\
Z_{\theta}^{(m)}
\end{array}\right\}(\omega, r, \theta)=\tilde{Z}_{T}^{(m)}(k)\left(\frac{r}{a_{1}}\right)^{m-1}\left(\frac{r_{q}}{a_{1}}\right)^{m}\left\{\begin{array}{c}
\cos m \theta \\
-\sin m \theta
\end{array},\right.
\end{aligned}
$$

where $\omega$ is the frequency, $(r, \theta)$ are the polar coordinates, $k=\omega / v$ is the wave number, $a_{1}$ is the internal radius of the chamber with circular cross section. The frequency dependent part of impedances $\tilde{Z}_{\|}^{(m)}(k), \tilde{Z}_{T}^{(m)}(k)$ are coupled by PanowskyWenzel theorem as $\tilde{Z}_{T}^{(m)}=m \tilde{Z}_{\|}^{(m)} /\left(k a_{1}\right)$ [2]. The dimensions of the longitudinal and transverse impedances per unit length are $\mathrm{Ohm} / \mathrm{m}$. For the multi-layered vacuum chamber the impedances can be evaluated using the field matching technique [2], i.e. the continuity of tangential components of electric and magnetic fields on the layers boarders, and introducing the field transformation matrix $\hat{Q}$ for tangential components of the fields [4,5]. The longitudinal impedance can be then expressed via the elements $Q_{i j}$ of field transformation matrix $\hat{Q}(\operatorname{dim} 4 \times 4)$ as 


$$
\tilde{Z}_{\|}^{(m)}(k)=\left\{\begin{array}{c}
j \frac{Z_{0}}{\pi k a_{1}^{2}}\left(1-\frac{2}{k a_{1}} \frac{Q_{33}}{Q_{23}}\right)^{-1} \text { for } m=0 \\
j \frac{Z_{0}}{\pi} k\left(\frac{k^{2} a_{1}^{2}}{m+1}-m+\frac{k a_{1} V_{m}^{+}}{V_{m}^{-}}\right)^{-1} \text { for } m>0
\end{array}\right.
$$

The field transformation matrix $\hat{Q}$ couples the tangential components of the electric and magnetic fields at the inner $\left(r=a_{1}\right)$ and outer $\left(r=a_{N+1}\right)$ surfaces of the pipe and for $N$ layer tube is calculated as

$$
\hat{Q}=\widehat{Q}^{(1)} \widehat{Q}^{(2)} \cdots \widehat{Q}^{(N)},
$$

where $\hat{Q}^{(i)}$ is the field transformation matrix through the $i$-th layer $(i=1,2,3 \ldots N)$

$$
\hat{Q}^{(i)}=\left(\begin{array}{cccc}
q_{11} & q_{12} & -\alpha_{0} q_{31} & \alpha_{0} q_{32} \\
0 & q_{22} & -\alpha_{0} q_{41} & \alpha_{0} q_{42} \\
q_{31} & q_{32} & q_{11} & -q_{12} \\
q_{41} & q_{42} & 0 & q_{22}
\end{array}\right)
$$

with $\alpha_{0}=\varepsilon_{i}^{\prime} / \mu_{i}^{\prime}, \quad \varepsilon_{i}^{\prime}=\varepsilon_{i} / \varepsilon_{0}, \mu_{i}^{\prime}=\mu_{i} / \mu_{0}$, the relative dielectric and magnetic permeabilities of each layer with respect to the vacuum constants $\varepsilon_{0}, \mu_{0}$. The seven independent elements of the field transformation matrix $\hat{Q}^{(i)}$ are determined by corresponding geometrical and electromagnetic layer parameters and are:

$$
\begin{aligned}
& q_{11}=-a_{i+1} \chi_{i} U_{3}^{(i)}, q_{22}=a_{i+1} \chi_{i} U_{2}^{(i)}, \\
& q_{12}=\frac{j k m}{a_{i} \chi_{i}^{2}}\left(\frac{a_{i}}{a_{i+1}} q_{11}-q_{22}\right), q_{31}=\frac{j a_{i+1}}{\mu_{i}^{\prime} a_{i}} m U_{1}^{(i)}, \\
& q_{32}=k\left(\beta^{2} \varepsilon_{i}^{\prime} a_{i+1} U_{4}^{(i)}+\frac{m^{2} U_{1}^{(i)}}{\mu_{i}^{\prime} a_{i} \chi_{i}^{2}}\right), \\
& q_{41}=-\frac{a_{i+1} \chi_{i}^{2}}{k \mu_{i}^{\prime}} U_{1}^{(i)}, \quad q_{42}=\frac{a_{i+1}}{a_{i}} q_{31},
\end{aligned}
$$

where $\chi_{i}=\sqrt{k^{2}-\mu_{i} \varepsilon_{i} \omega^{2}} \quad\left(\operatorname{Re}\left(\chi_{i}\right)>0\right)$ are the radial propagation constants. The functions $U_{k}^{(i)}(k=1,2,3,4)$ are the combinations of modified Bessel functions of both kinds. In the frequency range, the functions $U_{k}^{(i)}$ can be approximated by hyperbolic functions if the skin depth of a layer is much smaller than the inner radius of the tube, i.e. for all practically important cases: 


$$
\begin{aligned}
& U_{1}^{(i)}=K_{m}\left(\chi_{i} a_{i}\right) I_{m}\left(\chi_{i} a_{i+1}\right)-I_{m}\left(\chi_{i} a_{i}\right) K_{m}\left(\chi_{i} a_{i+1}\right) \approx \operatorname{Sinh}\left(\chi_{i} d_{i}\right) / \chi_{i} \sqrt{a_{i+1} a_{i}}, \\
& U_{2}^{(i)}=K_{m}\left(\chi_{i} a_{i}\right) I_{m}^{\prime}\left(\chi_{i} a_{i+1}\right)-I_{m}\left(\chi_{i} a_{i}\right) K_{m}^{\prime}\left(\chi_{i} a_{i+1}\right) \approx \operatorname{Cosh}\left(\chi_{i} d_{i}\right) / \chi_{i} \sqrt{a_{i+1} a_{i}}, \\
& U_{3}^{(i)}=K_{m}^{\prime}\left(\chi_{i} a_{i}\right) I_{m}\left(\chi_{i} a_{i+1}\right)-I_{m}^{\prime}\left(\chi_{i} a_{i}\right) K_{m}\left(\chi_{i} a_{i+1}\right) \approx-\operatorname{Cosh}\left(\chi_{i} d_{i}\right) / \chi_{i} \sqrt{a_{i+1} a_{i}}, \\
& U_{4}^{(i)}=K_{m}^{\prime}\left(\chi_{i} a_{i}\right) I_{m}^{\prime}\left(\chi_{i} a_{i+1}\right)-I_{m}^{\prime}\left(\chi_{i} a_{i}\right) K_{m}^{\prime}\left(\chi_{i} a_{i+1}\right) \approx-\operatorname{Sinh}\left(\chi_{i} d_{i}\right) / \chi_{i} \sqrt{a_{i+1} a_{i}} .
\end{aligned}
$$

In (6) $d_{i}=a_{i+1}-a_{i}$ is the thickness of the $i$-th layer. All elements of the matrix $\hat{Q}^{(i)}$ (4) are dimensionless and the determinant of the matrix is equal to $a_{i+1}^{2} / a_{i}^{2}$. As is seen from (5), for the monopole term $m=0$ the following elements of field transformation matrix $q_{12}=q_{13}=q_{21}=q_{24}=q_{31}=q_{34}=q_{42}=q_{43} \equiv 0$ are vanishing.

The other coefficients in (2) are determined as

$$
\begin{array}{ll}
V_{m}^{ \pm}=k a_{N+1} A^{ \pm}+\left(1+2 k^{2} a_{N+1}^{2} P_{0}\right) B^{ \pm} & \text {for } \quad m=1, \\
V_{m}^{ \pm}=(m-1) k a_{N+1} A^{ \pm}+\left[m(m-1)-k^{2} a_{N+1}^{2}\right] B^{ \pm}, & \text {for } \quad m>1,
\end{array}
$$

with $P_{0}=\ln \left(k a_{N+1} / 2 \gamma\right)+C_{E}, C_{E}=0.577216$ the Euler constant, $\gamma$ the particle Lorentz factor . Coefficients $A^{ \pm}, B^{ \pm}$are defined via the elements of field transformation matrix

$$
\begin{aligned}
& A^{+}=\operatorname{Det}\left[\widehat{h}_{11 ; 22}+\widehat{h}_{33 ; 44}+j\left(\widehat{h}_{13 ; 24}-\widehat{h}_{31 ; 42}\right)\right], \\
& A_{m}^{-}=\operatorname{Det}\left[\hat{h}_{21 ; 42}+j \hat{h}_{23 ; 44}\right] \\
& B^{+}=\operatorname{Det}\left[\widehat{h}_{11 ; 23}-j \widehat{h}_{31 ; 43}\right] \\
& B_{m}^{-}=\operatorname{Det}\left[\widehat{h}_{21 ; 43}\right]
\end{aligned}
$$

where the matrix $\hat{h}_{p i ; q j}$ is composed as

$$
\widehat{h}_{p i ; q j}=\left\{\begin{array}{ll}
Q_{p i} & Q_{p j} \\
Q_{q i} & Q_{q j}
\end{array}\right\},
$$

Note that in ultra-relativistic limit $(\ln \gamma \rightarrow \infty)$ for dipole mode $(m=1)$, $U^{+} / U^{-}=B^{+} / B^{-}$.

\subsubsection{Impedance of Vacuum Chamber with Elliptical Cross Section}

In the case of a elliptical vacuum chamber the impedance significantly depends on the transverse position of the beam. We consider the practically important case when the beam has a horizontal or a vertical offset with respect to ellipse center. We will evaluate the impedances for an elliptical vacuum chamber following the approach developed in Ref. [6] that generalize the round chamber impedances for elliptical cross section by introducing longitudinal and transverse geometrical correction factors. For the elliptical geometry with major $a$ and minor $b$ radii the longitudinal and transverse impedances may be expressed then via the round chamber impedances with radius $b$ 
(ellipse minor radius) multiplied by the longitudinal $G_{\|}$or transverse $G_{v}$ (vertical), $G_{h}$ (horizontal) geometrical factors:

$$
Z_{\|}(\omega)=Z_{s}^{0}(\omega) G_{s}, \quad Z_{v, h}(\omega)=Z_{t}^{0}(\omega) G_{v, h}
$$

The geometrical factors depend on the ellipse aspect ratio $a / b$ and are defined as

$$
\begin{aligned}
& G_{s}(\omega)=\frac{1}{2 \pi} \int_{0}^{2 \pi} \frac{f_{c}^{2}(\xi)}{g(\xi)} d \xi \\
& G_{v, h}(\omega)=\frac{1}{2 \pi\left(a^{2} / b^{2}-1\right)} \int_{0}^{2 \pi} \frac{f_{c}(\xi) f_{t}(\xi)+2 f_{v, h}^{2}(\xi)}{g(\xi)} d \xi
\end{aligned}
$$

where

$$
\begin{aligned}
& f_{c}(\xi)=1+2 \sum_{m=1}^{\infty} \frac{\cos m \xi}{c h \eta_{0}} \cos \frac{m \pi}{2}, \quad f_{t}(\xi)=\sum_{m=1}^{\infty} m^{2} \frac{\cos m \xi}{c h \eta_{0}} \cos \frac{m \pi}{2} \\
& f_{v}(\xi)=\sum_{m=1}^{\infty} m \frac{\sin m \xi}{\sinh m \eta_{0}} \sin \frac{m \pi}{2}, \quad f_{h}(\xi)=-\sum_{m=1}^{\infty} m \frac{\cos m \xi}{\cosh m \eta_{0}} \sin \frac{m \pi}{2} \\
& g(\xi)=\sqrt{1+\sin ^{2} \xi / \sinh ^{2} \eta_{0}}, \quad \eta_{0}=\frac{1}{2} \ln \frac{1+b / a}{1-b / a}
\end{aligned}
$$

with $(\xi, \eta)$ the elliptical coordinates.

The geometrical factor for the longitudinal impedance is equal to unity $\left(G_{s}=1\right)$ for a round tube $(a / b=1)$ and closed to unity for large values of $a / b: G_{s} \rightarrow 1$ for $a / b \rightarrow \infty$, i.e. the longitudinal impedance of the round tube of radius $b$ coincides with the impedance of a flat chamber with a distance of $2 b$ between plates [7]. For the transverse impedance: for a large aspect ratio $a / b$ the horizontal geometrical factor drops while the vertical geometrical factor approaches to $\pi^{2} / 8 \approx 1.23$. 

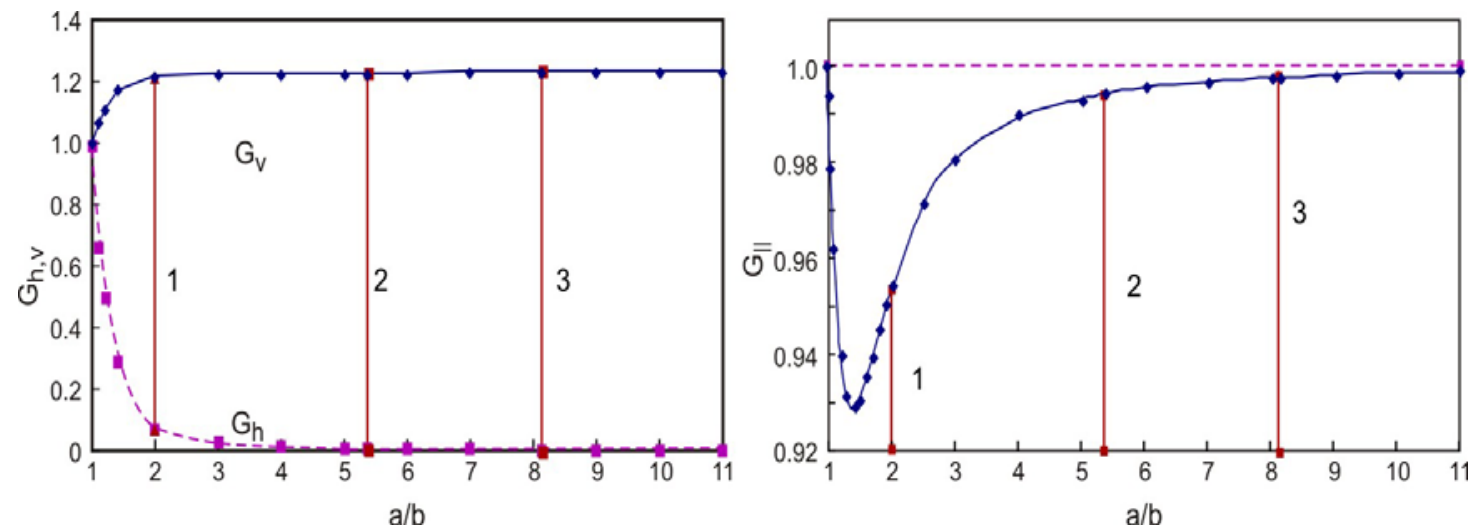

Figure 1: The longitudinal (left) and transverse (right) geometrical factors vs aspect ratio $a / b$. Standard arc (1), wiggler (2) and undulator (3) chambers.

Table 2 shows the correction factors for the PETRA III vacuum chambers with elliptical cross section. As is seen, the transverse impedance for elliptical vacuum chambers is actually dominated by the vertical impedance.

Table 2: Geometrical factors for PETRA III elliptical vacuum chambers.

\begin{tabular}{l|c|c|c}
\hline & Standard Arc & Wiggler & Undulator \\
\hline Aspect ratio $a / b$ & 2 & 5.36313 & 8.14286 \\
\hline Longitudinal $\mathrm{G}_{\|}$ & 0.9531 & 0.9941 & 0.9975 \\
\hline Vertical $\mathrm{G}_{\mathrm{v}}$ & 1.2213 & 1.2322 & 1.2330 \\
\hline Horizontal $\mathrm{G}_{\mathrm{h}}$ & 0.0764 & 0.0088 & 0.0038 \\
\hline
\end{tabular}

In the current paper the impedances in all the figures are evaluated for the round chamber geometry. For the vacuum chambers with elliptical cross-section, the transverse vertical impedance should be multiplied by the geometrical factor of $\pi^{2} / 8$.

\subsubsection{Impedance of Finite Thickness Single -Layer Vacuum Chamber}

For the single layer vacuum chamber of internal radius $a$ and finite wall thickness $d$ the monopole $(m=0)$ and dipole $(m=1)$ modes of the impedance in ultrarelativistic limit for non-magnetic wall $\left(\mu_{1}=\mu_{0}\right)$ can be presented in explicit form as

$$
\tilde{Z}_{\|}^{(m)}(k)=\left\{\begin{array}{c}
j \frac{Z_{0}}{\pi k a^{2}}\left(1+\frac{2}{a \chi \varepsilon^{\prime}} \frac{U_{3}}{U_{1}}\right)^{-1} \text { for } m=0 \\
j \frac{Z_{0} k}{\pi}\left[\frac{k^{2} a^{2}}{2}-\frac{\varepsilon^{\prime}+1}{\varepsilon^{\prime}-1}\left(1-a \chi \frac{U_{3}}{U_{1}}\right)\right]^{-1} \text { for } m=1
\end{array}\right.
$$

If the skin depth of the layer is much smaller than the inner radius of the tube the functions $U_{1}, U_{3}$ are approximated by hyperbolic functions according to (6) and $U_{3} / U_{1} \approx c t h(\chi d)$ with $d$ the wall thickness. For the infinite wall thickness $(d \rightarrow \infty)$ 
and commonly used approximation ( $\omega<<\sigma / \varepsilon_{0}, \sigma$ - conductivity) formula (15) is converted to well-known presentation of the impedance $[1,2]$ :

$$
\tilde{Z}_{\|}^{(0,1)}=h_{0,1} \frac{Z_{0} s_{0}}{2 \pi a^{2}}\left(\frac{1+j}{\sqrt{k_{0}}}-\frac{j k_{0}}{2}\right)^{-1},
$$

where $h_{0}=1$ for the monopole term, $h_{1}=2$ for the dipole term, $s_{0}=\left(2 a^{2} / Z_{0} \sigma\right)^{1 / 3}$ is the characteristic distance and $k_{0}=k s_{0}$ is the dimensionless wave-number.

\subsubsection{Standard Straight Section}

Figures 2 and 3 present the longitudinal and transverse multipole impedances for the PETRA III standard straight section vacuum chamber with circular cross section (see Table 1) based on the exact solution given by (2).
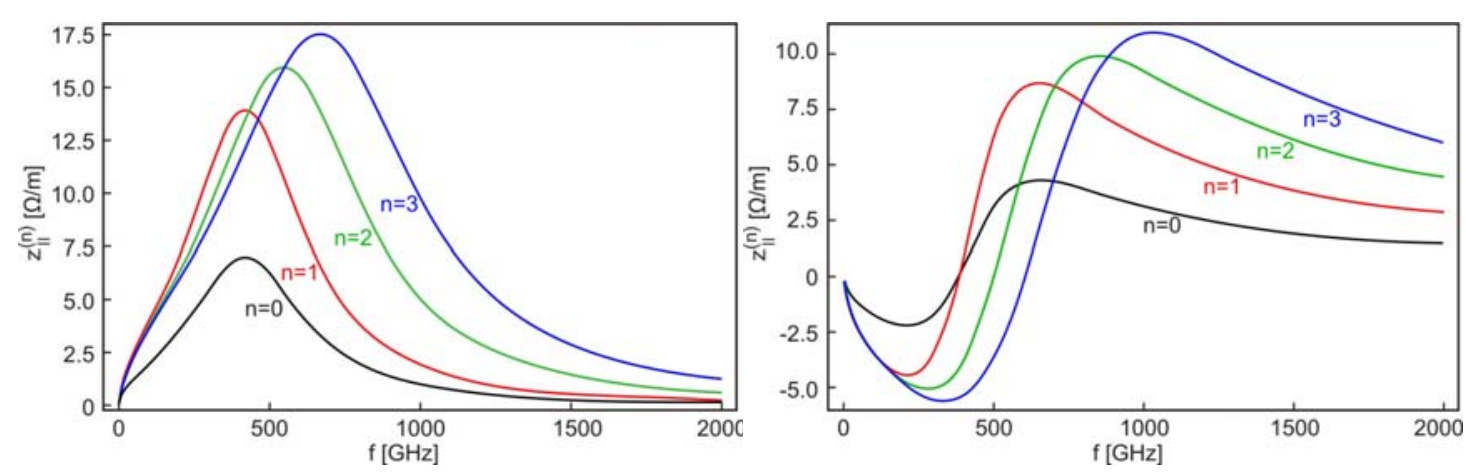

Figure 2: Real (left) and imaginary (right) parts of the longitudinal impedance for a standard straight section. Tube material- stainless steel, inner radius - $47 \mathrm{~mm}$, wall thickness - $2 \mathrm{~mm}$.
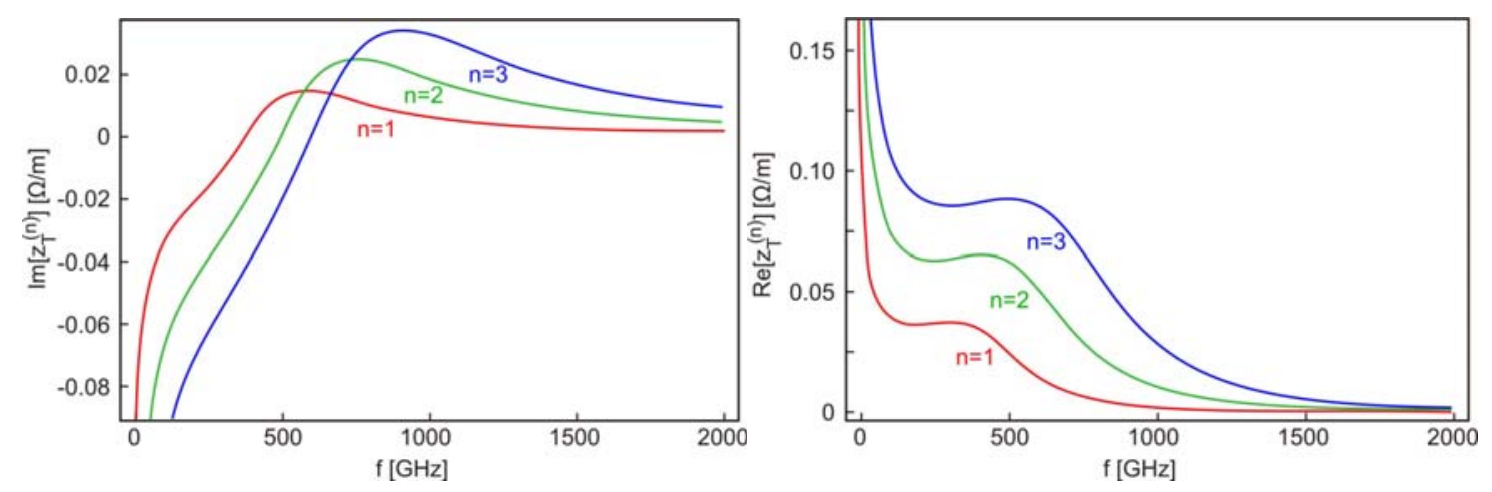

Figure 3: Real (left) and imaginary (right) parts of the transverse impedance for a standard straight section. Tube material- stainless steel, inner radius - $47 \mathrm{~mm}$, wall thickness - $2 \mathrm{~mm}$.

For a wall thickness of $2 \mathrm{~mm}$ the skin depth is equal to the wall thickness when the frequency is equal to $f_{0} \sim 42 \mathrm{kHz}$. For frequencies $f>>f_{0}$ the results are well described by the general approximation (16) for infinite wall thickness. 
The difference arises for the frequencies $f \leq f_{0}$. Figures 4 and 5 show the real and imaginary parts of the longitudinal monopole and transverse dipole impedances in $\mathrm{kHz}$ frequency region for the exact solution and the approximation (16).
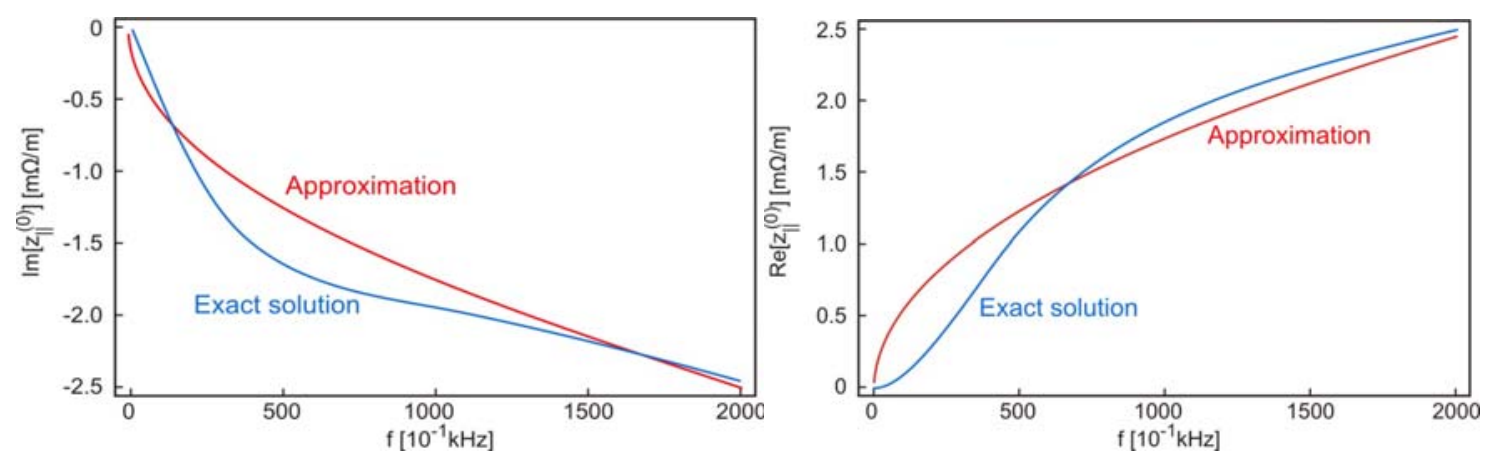

Figure 4: Real and imaginary parts of the longitudinal monopole impedance for the exact solution and the infinite thickness approximation in the $\mathrm{kHz}$-frequency region.
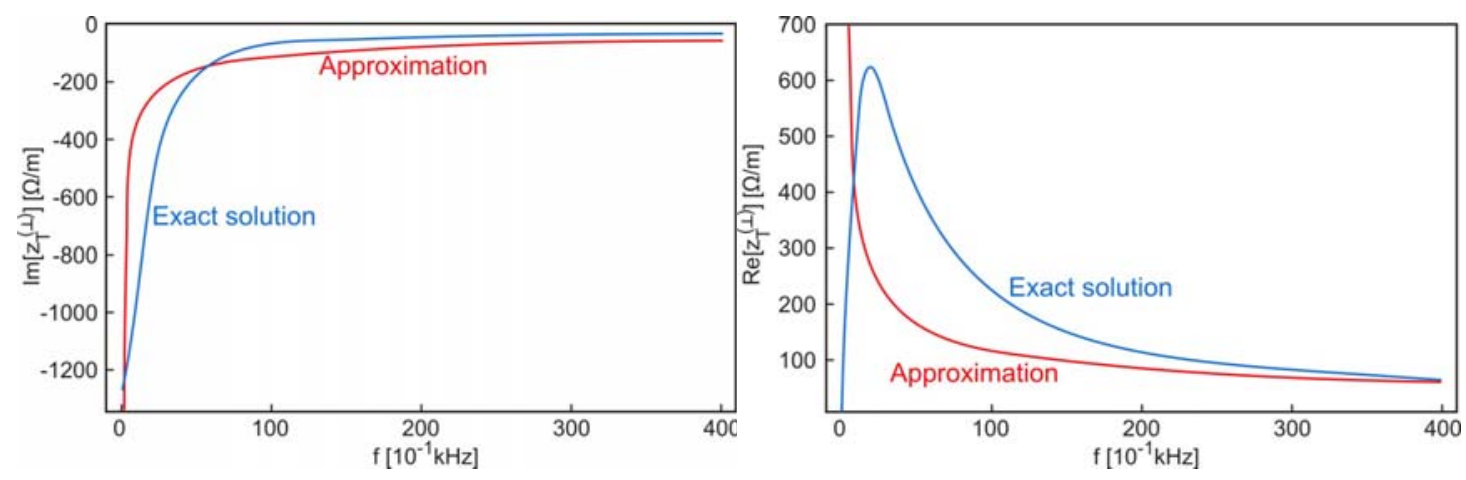

Figure 5: Real and imaginary parts of the transverse dipole impedance for the exact solution and the infinite thickness approximation in the kHz-frequency region.

As one can see from Figure 5, the transverse impedance given by the approximation (16) diverges at $\omega=0$, while the exact solution gives $Z_{T}^{(m)}(\omega=0)=-j Z_{0} / 2 \pi a$ for all multipoles and does not depend on the the material and the thickness of the chamber wall.

\subsubsection{Frequency-Dependent Conductivity.}

The frequency dependent conductivity of the metal is given by

$$
\sigma(\omega)=\sigma_{s t}(1-j \omega \tau)^{-1}
$$

where $\sigma_{\text {st }}$ is the static conductivity and $\tau$ is the relaxation time. A comparison of the longitudinal monopole impedance of the stainless steel tube with a frequency dependent (AC) and a frequency independent (DC) conductivity is presented in Fig. 6. The relaxation time is taken to be $\tau=3.2 \cdot 10^{-14} \mathrm{sec}$, which corresponds to the relaxation time of iron at a temperature of $T=77 \mathrm{~K}$.

The transverse dipole impedance for the same vacuum chamber with AC and DC conductivity is presented in Figure 7. As is seen from the results the impedances actually coincide for the given relaxation time. 

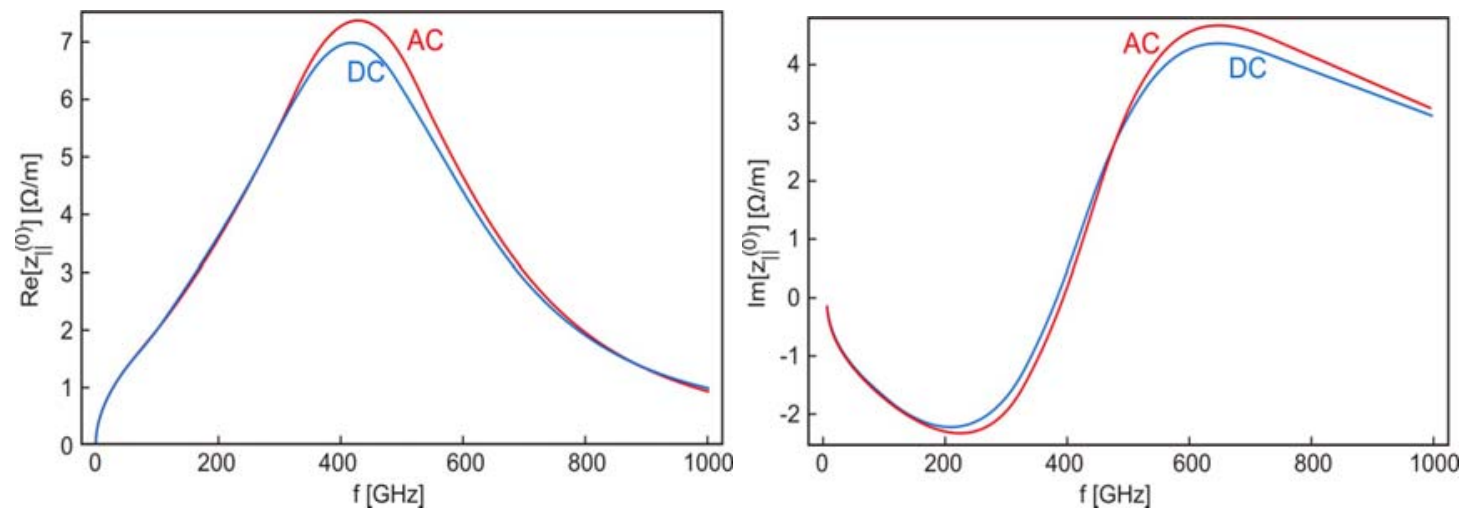

Figure 6: Longitudinal monopole impedance of a standard vacuum chamber for DC and AC conductivities.
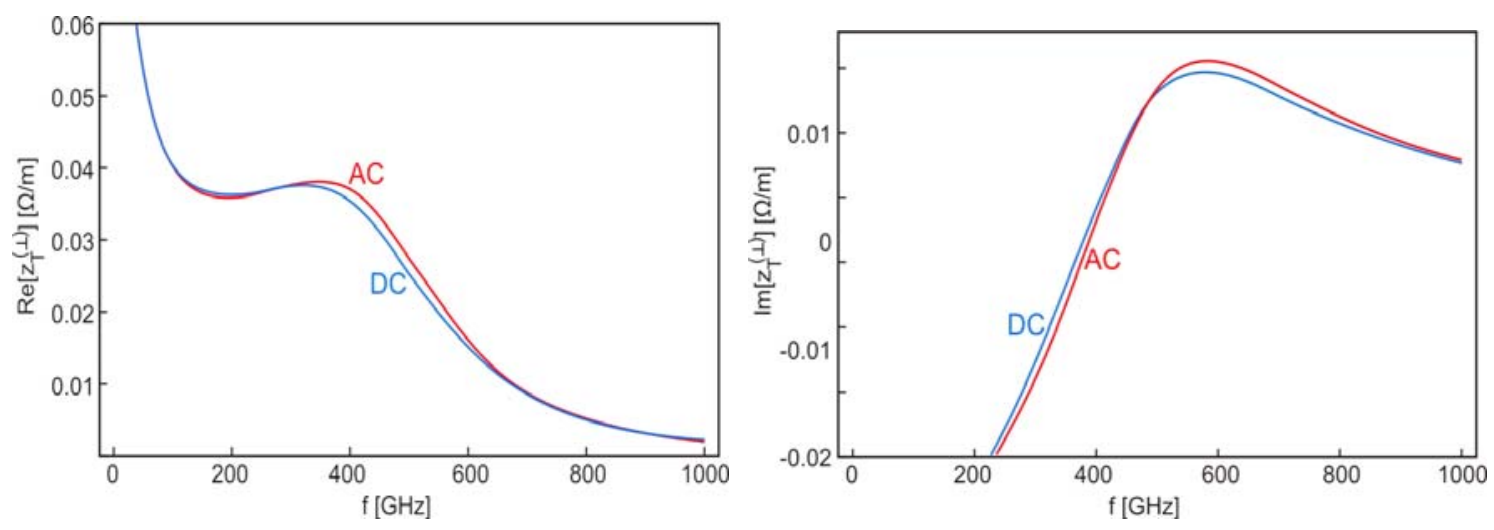

Figure 7: Transverse dipole impedance of a standard vacuum chamber for DC and AC conductivities.

\subsubsection{Undulator Vacuum Chamber}

The vacuum chamber of the undulator is an aluminium chamber with elliptical cross section and a wall thickness of $1 \mathrm{~mm}$. Figure 8 presents the real and imaginary parts of the transverse dipole impedance. 


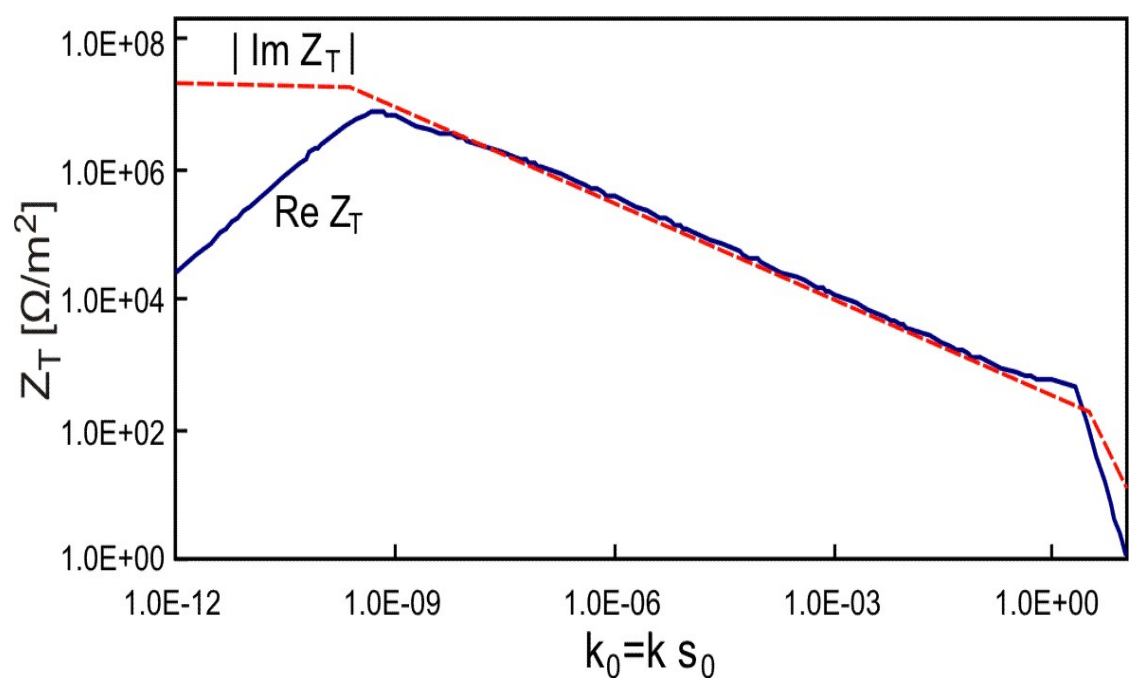

Figure 8: Transverse impedance of undulator vacuum chamber.

\subsubsection{Impedance of Two-Layer Vacuum Chamber}

The technical solutions for the vacuum chambers of the wiggler magnets and injection kickers in the PETRA III storage ring imply the usage of laminated chamber configurations. The wiggler magnet chamber is an aluminum chamber of elliptical cross section with NEG (Non-Evaporable Getter) coating on the inner side of the wall. The injection kicker vacuum chamber is a ceramic chamber of circular cross section covered with Titanium-Stabilized High Gradient Steel (TSHGS), a special metal alloy.

\subsubsection{Wiggler Vacuum Chamber}

Fig. 9 presents the real and imaginary parts of the transverse dipole impedance for a wiggler aluminum vacuum chamber coated with NEG material of $0.5 \mu \mathrm{m}$ and $1 \mu \mathrm{m}$ thickness. For a NEG thickness of $1 \mu \mathrm{m}$, the frequency that corresponds to the skin depth equal to cover thickness is in the order of $f_{0} \sim 820 \mathrm{GHz}$. For this case, as it can be seen from Fig. 9, the impact of the NEG-coating is visible for frequencies $f \geq 5-10 \mathrm{GHz}$.
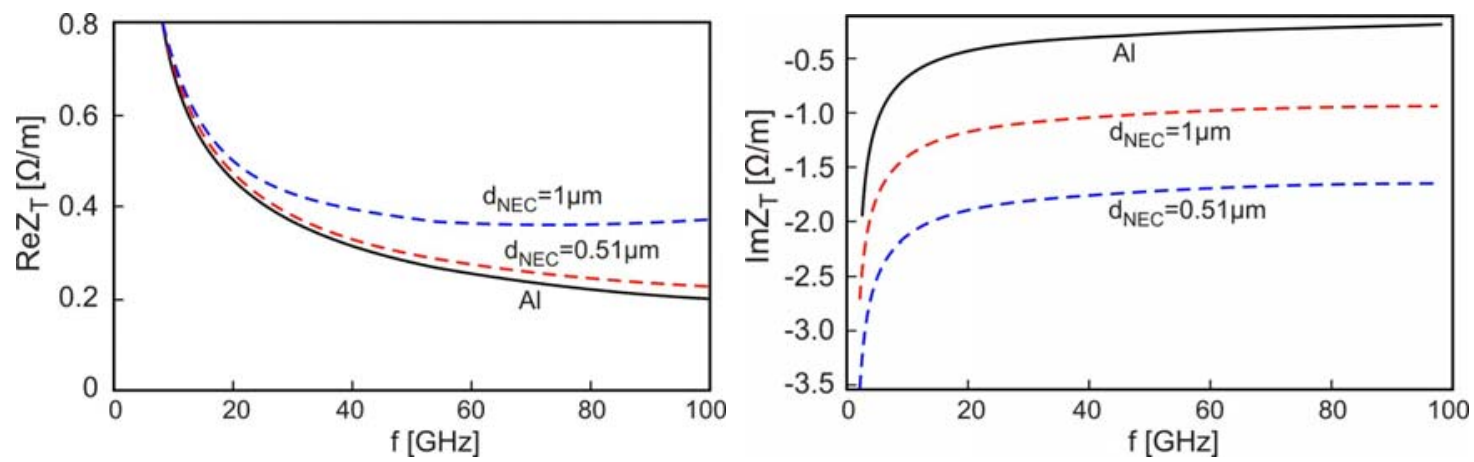

Figure 9: Real and imaginary parts of the transverse dipole impedance for the PETRA III wiggler vacuum chamber coated with NEG material. 
Figure 10 presents the dependence of the kick factor $k_{T}$ and integrated gradient of longitudinal wake $k_{I I}(1)$ produced by the wiggler vacuum chamber versus the NEG thickness.

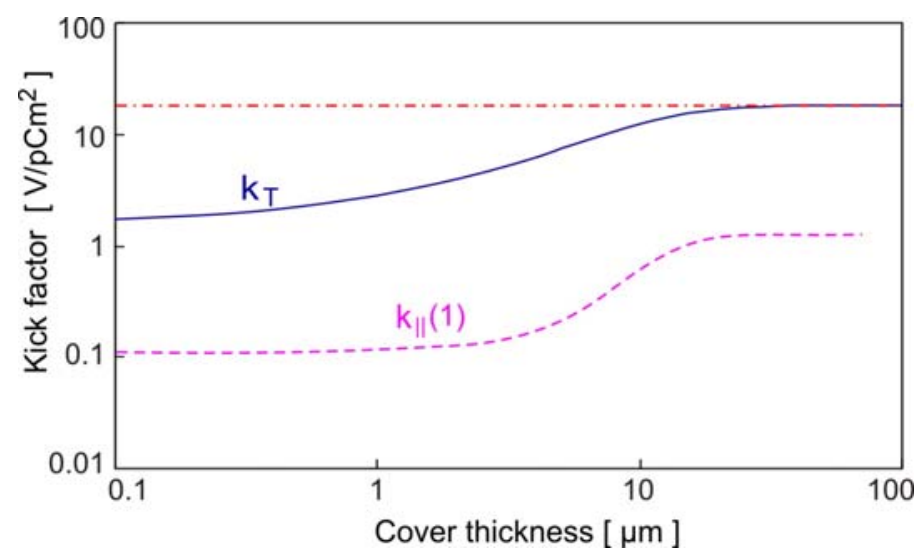

Figure 10: Kick factor (solid) and integral gradient of the longitudinal wake (dashed) versus cover thickness. The long-range approximation is plotted as well.

\subsubsection{Injection Kicker}

The vacuum chamber of the PETRA III injection kicker is a round ceramic tube with thickness $1 \mathrm{~cm}$, which is covered at the inner side with Titanium-Stabilized High Gradient Steel (TSHGS), a special metal alloy. The layer thickness of the TSHGS is equal to $0.7 \mu \mathrm{m}$. The relative electrical permitivity of the ceramic material is taken as $\varepsilon_{r}=9.1$ with a loss tangent of $\tan \delta \sim 10^{-4}$, so that the absolute electrical permitivity is equal to $\varepsilon_{c e r}=\varepsilon_{r} \varepsilon_{0}(1-j \tan \delta) A s / V m$. The cover conductivity is in the order of $\sigma_{\text {cover }}=2.0841 \cdot 10^{6} \Omega^{-1} m^{-1}$.

Figure 11 presents the transverse dipole impedance for the injection kicker vacuum chamber which is compared with the impedance of ceramic tube and the impedance of a pure TSHGS vacuum chamber. As seen from Figure 11, the ceramic tube impedance in the low frequency region has a constant value and is characterized by the narrowmeshed periodical oscillations. It should be noted that the oscillations amplitude decreases with the increasing of the wall thickness. The fine structure of ceramic tube impedance is characterized by narrow-band resonances (Fig. 12, left). 


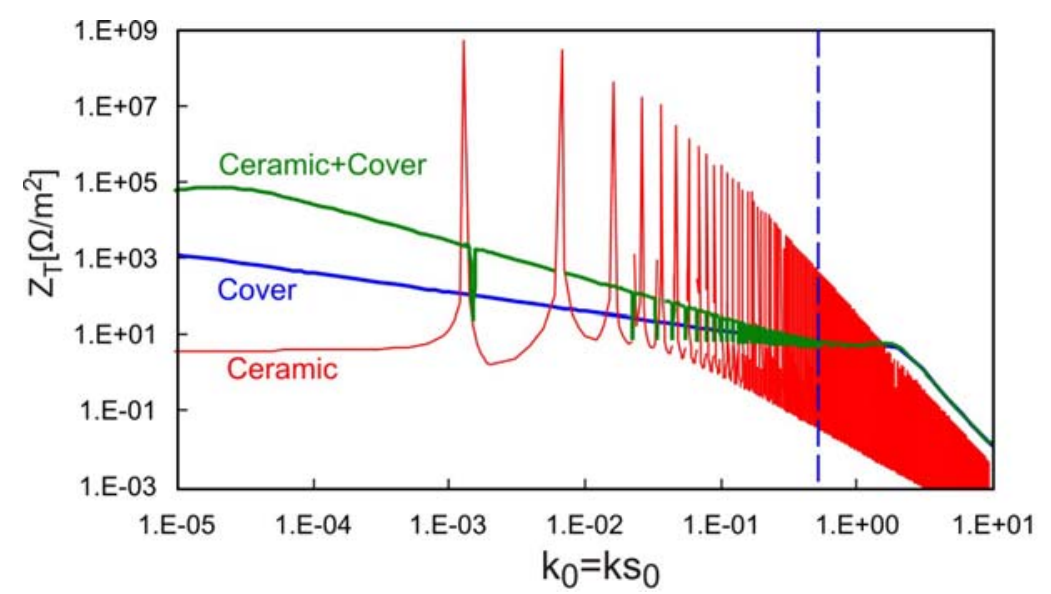

Figure 11: Injection kicker transverse impedance (real part).
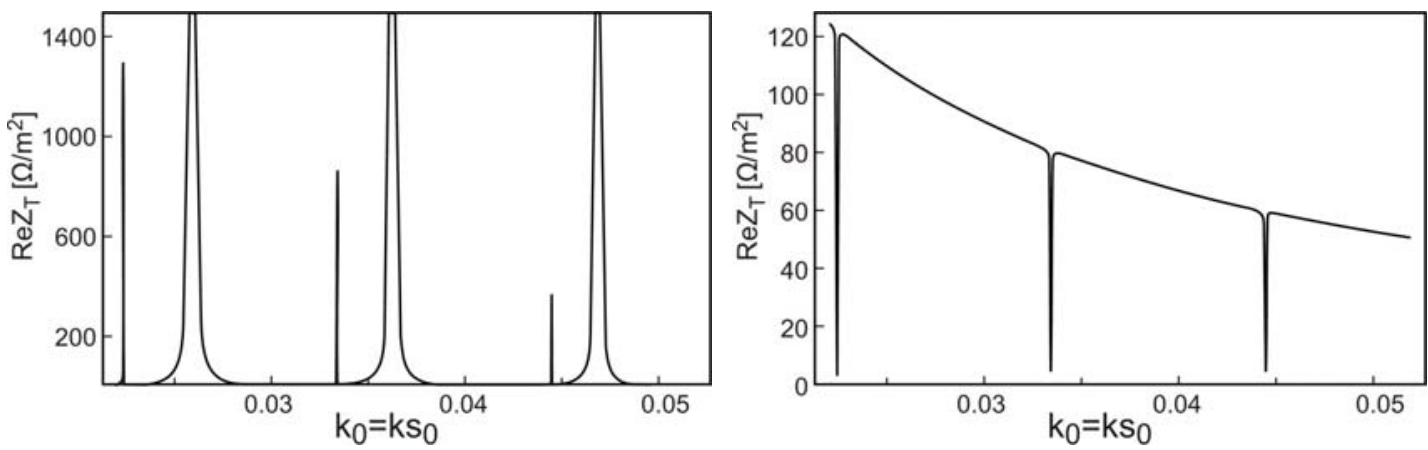

Figure 12: Fine structure of the transverse impedance for a ceramic (left) and a ceramic + TSHGS (right) vacuum chamber.

The impedance of a covered ceramic tube can be interpreted as a smooth curve: the high-level resonances disappear and the low-level notches appear instead of narrowband low-level resonances of ceramic tube impedance (Fig. 12, right). In the high frequency region, then the skin depth of the cover material surpasses the thickness of the cover layer $\left(k_{0}>0.52\right)$ and the impedance of the ceramic-metal tube is fully determined by metallic layer material.

The kick factor of the injector kicker depends weakly on thickness of the ceramic layer if the layer is larger than $2.5 \mathrm{~cm}$, as shown in Fig. 13.

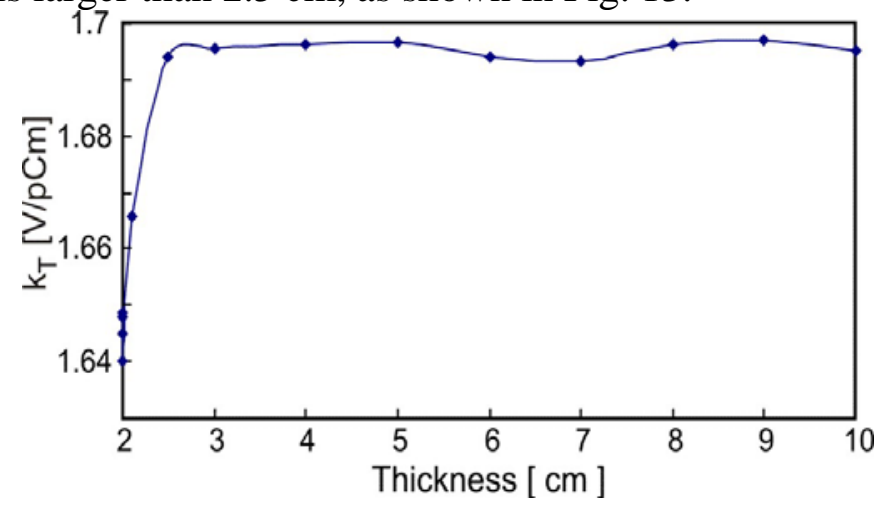

Figure 13: Kick parameter versus ceramic wall thickness. 


\subsubsection{Transverse Kick Factor and Integral Gradient}

The instability threshold for mode coupling instabilities can be estimated from the tune shifts $\Delta Q_{s}, \Delta Q_{\beta}$ of the lowest order modes in the longitudinal and transverse planes [8]:

$$
\begin{aligned}
\Delta Q_{\beta} & =\frac{I_{B}\langle\beta\rangle T_{0}}{4 \pi E / e} k_{\perp}, \\
\Delta Q_{s} & =Q_{s} \frac{I_{B} R T_{0}}{2 h V_{r f}} k_{\|}(1),
\end{aligned}
$$

where $k_{\perp}$ is the transverse kick factor, $k_{\|}(1)$ is the integral gradient of longitudinal wake, $I_{B}$ is the single bunch current, $R=367 \mathrm{~m}$ is the storage ring mean radius, $\langle\beta\rangle$ is the average beta function, $E$ is the particle energy, $T_{0}$ is the revolution period, $V_{r f}$ is RF gap voltage and $h$ is the harmonic number. The following tolerable tune shifts are assumed

$$
\frac{\Delta Q_{\beta}}{Q_{s}}=0.5, \quad \frac{\Delta Q_{s}}{Q_{s}}=0.5
$$

That determines the maximal acceptable values for total integrated gradient $k_{\|}(1)$ and kick factor $k_{\perp}$ in the ring. The integral gradient $k_{\|}(1)$ and kick factor $k_{\perp}$ of the Gaussian bunch with rms length $\sigma_{z}$ are defined by the monopole and dipole impedances as

$$
\begin{aligned}
& k_{\|}(1)=\left|\frac{1}{\pi} \int_{0}^{\infty} d \omega k \operatorname{Im}\left[Z_{\|}^{(0)}(\omega)\right] \exp \left(-k^{2} \sigma_{z}^{2}\right)\right| \\
& k_{T}=\frac{1}{\pi a} \int_{0}^{\infty} d \omega \operatorname{Im}\left[\tilde{Z}_{T}^{(1)}(\omega)\right] \exp \left(-k^{2} \sigma_{z}^{2} /\right) .
\end{aligned}
$$

The total integral gradient of the longitudinal wake and the kick factor of the ring are the sum of the contributions from all vacuum chamber components (wigglers, undulators, standard sections)

$$
k_{\|}(1)_{\text {total }}=\sum_{i} k_{\|}(1)_{i} L_{i}, \quad k_{\perp t o t a l}=\frac{1}{\langle\beta\rangle} \sum_{i} \beta_{i} k_{\perp i} L_{i}
$$

where $\beta_{i}$ is the local beta function ( $\beta_{y}=5 \mathrm{~m}$ for undulator section, $\beta_{y}=15 \mathrm{~m}$ for wiggler section and $\beta_{y}=20 \mathrm{~m}$ for the standard arc), $\langle\beta\rangle$ is the average beta function ( $\langle\beta\rangle=20 m), L_{i}$ is the length of the corresponding vacuum chamber path. 
Table 3: The integrated gradient and kick factor.

\begin{tabular}{l|c|c|c|c}
\hline \multicolumn{1}{c|}{ Device } & $\begin{array}{c}\beta_{y} \\
(\mathbf{m})\end{array}$ & $\begin{array}{c}\text { Length } \\
(\mathbf{m})\end{array}$ & $\begin{array}{c}\mathbf{k}_{\text {|l }}(\mathbf{1}) \\
(\mathbf{V} / \mathbf{p C} / \mathbf{m})\end{array}$ & $\begin{array}{c}\mathbf{k}_{\perp} \\
(\mathbf{V} / \mathbf{p C} / \mathbf{m})\end{array}$ \\
\hline Undulator & 5 & 55 & 15.57 & 450.8 \\
\hline Wiggler & 15 & 80 & 8.45 & 203.4 \\
\hline Arc & 20 & 1411 & 68.12 & 247.6 \\
\hline Total & - & 1546 & 91.14 & 901.8 \\
\hline
\end{tabular}

Table 3 presents the resistive wall contributions to the longitudinal and transverse impedance budget of PETRA III (in terms of $k_{\|}(1)$ and $k_{\perp}$ ) for a bunch length of $\sigma_{z}=12 \mathrm{~mm}$. The total integral gradient of longitudinal wake is estimated to be $k(1)_{\| \text {total }}=91.14 \mathrm{~V} / \mathrm{pCm}$ and the total kick factor as $k_{\perp \text { total }}=902 \mathrm{~V} / \mathrm{pC} / \mathrm{m}$. Thus the longitudinal and transverse resistive wall contributions to the total tolerable impedance budget are $0.87 \%$ and $15.3 \%$ respectively.

\subsubsection{Conclusion}

In the present paper the longitudinal and transverse resistive impedances for different parts of PETRA III storage ring are evaluated. The impedances for single layer finite thickness and two-layer vacuum chambers are evaluated for both circular and elliptical geometry of the chamber. The contribution of the resistive wall impedance in terms of the kick factor and the integral gradient of the longitudinal wake to the total impedance budget have been estimated.

\subsubsection{Acknowledgment}

The authors would like to express thanks to Klaus Balewski, Winfried Decking and Vitali Khachatryan for many stimulating discussions.

\subsubsection{References}

1. "PETRA III: A low Emittance Synchrotron Radiation Source", Technical Design Report, DESY 2004-035; http://petra3.desy.de/project_description

2. B.W.Zotter and S. A. Kheifetz, Impedances and Wakes in High-Energy Particle Accelerators, World Scientific, Singapore, 1997.

3. A.W. Chao, Physics of Collective Beam Instabilities in High Energy Accelerators (Wiley, New York, 1993).

4. M. Ivanyan et al, Investigation of the PETRA III Resistive Wall Impedance, DESY M 07-01, September 2007.

5. M. Ivanyan et al, Phys. Rev. ST-AB ( to be published).

6. A. Piwinski, Report No. DESY HERA 92-11, 1992, p. 19.

7. H. Henke, O. Napoly, In Proc. of the $2^{\text {nd }}$ European Particle Accelerator Conference, Nice, France, pp. 1046-1048, 1990.

8. K. Balewski and R. Wanzenberg, Proceedings of 2005 Patricle Accelerator Conference, Knoxwill, Tennessee, USA, pp. 1751-1753, 2005. 